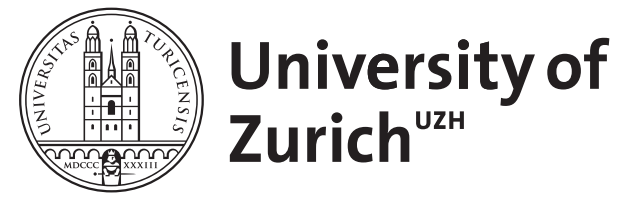

Zurich Open Repository and Archive

University of Zurich

University Library

Strickhofstrasse 39

CH-8057 Zurich

www.zora.uzh.ch

Year: 2012

\title{
Legionärskrankheit (Legionellen-Pneumonie)
}

Dobrick, Nadine ; Müller, Nicolas ; Franzen, Daniel

DOI: https://doi.org/10.1024/1661-8157/a001135

Posted at the Zurich Open Repository and Archive, University of Zurich

ZORA URL: https://doi.org/10.5167/uzh-71688

Journal Article

Originally published at:

Dobrick, Nadine; Müller, Nicolas; Franzen, Daniel (2012). Legionärskrankheit (Legionellen-Pneumonie). Praxis, 101(23):1459-1467.

DOI: https://doi.org/10.1024/1661-8157/a001135 


\section{CME}

\section{Legionärs-Krankheit (Legionellen-Pneumonie)}

Legionellen sind relativ häufige Erreger sowohl von „community-acquired“ als auch von „hospitalaquired“ Pneumonien, welche 1976 in der „American Legion Convention“ in Philadelphia erstmalig beschrieben wurden. Bisher sind 50 verschiedene Spezies der Legionella-Familie mit mehr als 70 Serogruppen bekannt. Legionella pneumonphila ist mit einem Vorkommen von $80 \%$ die häufigste Spezies, wobei nur die Serogruppen 1,4 und 6 für Humaninfektionen verantwortlich sind.

Die Legionärs-Krankheit (englisch: Legionnaire`s disease) ist eine relativ häufige Form der ambulantund spital-erworbenen Pneumonie und zählt zu den sogenannten „atypischen Pneumonien“. Sie steht an Platz drei bis vier der am häufigsten identifizierten Erreger bezüglich der ambulant erworbenen Pneumonie. Jedoch auch bei hospitalisierten Patienten sind die Legionellen ein gewichtiger Grund für schwer verlaufende Pneumonien. Daher sollte bei jedem Patienten mit der klinischen Diagnose einer schwer verlaufenden Pneumonie die Suche nach Legionellen erfolgen.

Ein häufig zitiertes Unterscheidungsmerkmal zwischen den so genannten atypischen und typischen Pneumonien ist das Vorhandensein respektive Fehlen von extrapulmonalen Symptomen, wobei atypische Pneumonien eher als Systemerkrankungen mit pulmonalen Symptomen gezählt werden. Eine Legionellen-Pneumonie präsentiert sich neben der Pneumonie-Symptomatik typischerweise mit hohem Fieber, gastrointestinalen Symptomen sowie Elektrolytstörungen.

Der primäre Abwehrmechanismus gegenüber Legionellen ist die zellvermittelte Immunität, wobei den Monozyten eine gesonderte Rolle zukommt. Defekte in der zellvermittelten Abwehr, wie z.B. eine Monozytenfunktionsstörung, führen zu einer erhöhten Anfälligkeit für Infektionen mit diesen Erregern. 
C. Klinik und Komplikationen

\section{Klinik}

Unter dem Begriff „Legionellose“ versteht man eigentlich zwei im Grund genommen unterschiedliche Erkrankungen, welche jedoch beide durch das Bakterium Legionella verursacht werden; die Legionärs-Krankheit ist eine durch Legionellen verursachte Pneumonie, während das Pontiac Fieber, eine eher gutartige, akute, febrile und in der Regel selbstlimitierende Erkrankung darstellt. In diesem Artikel widmen wir uns ausschliesslich der Legionärs-Krankheit.

Die Legionärs-Krankheit hat eine Inkubationszeit von 2 bis 14 Tagen, wobei die respiratorische Symptomatik initial nicht im Vordergrund stehen muss. Oft besteht nur ein leichter, kaum produktiver Husten, mit allenfalls leicht blutig tingiertem Sputum („minor“ Hämoptoe). Gastrointestinale Beschwerden äußern sich häufig als Diarrhoe, Übelkeit und Erbrechen sowie abdominalen Schmerzen. Des Weiteren präsentieren sich die Patienten häufig mit Kopfschmerzen und initialem Verwirrungszustand.

Patienten mit Legionärs-Krankheit haben typischerweise ab dem dritten Tag der Erkrankung konventionell radiologische pulmonale Veränderungen, wobei diese kein spezifisches Muster aufweisen. Häufig besteht ein monolobäres Infiltrat mit Konsoliderungsneigung, wobei die radiologischen Befunde dem klinischen Krankheitsverlauf zeitlich erst später folgen. Aus diesem Grund kann häufig eine Verschlechterung des radiologischen Befundes unter antibiotischer Therapie beobachtet werden. Auch bestehen radiologisch feststellbare Restveränderungen oft noch Monate nach der Erkrankung.

Laborchemische Veränderungen sind häufig und in der Regel unspezifisch. Sie beinhalten eine Erhöhung der Entzündungsparameter inklusive des Procalcitonins, eine Thrombozytopenie, eine renale und hepatische Dysfunktion sowie eine Hyponatriämie und Hypophosphatämie (siehe Tabelle 1). Ein Hyponatriämie kommt jedoch bei Pneumonien durch Legionellen häufiger vor als bei Pneumonien durch andere Erreger und sollte den Kliniker immer hellhörig werden lassen. Im Urinstatus bestehen häufig eine (Mikro-)Hämaturie und Proteinurie.

Zusammengefasst sollte bei einem Patienten mit Pneumonie und gastrointestinalen Symptomen (insbesondere Diarrhoe), neurologischen Auffälligkeiten, hohem Fieber $>39.0^{\circ} \mathrm{C}$, Hyponatriämie, Hepatopathie, (Mikro-)Hämaturie sowie fehlendem Ansprechen auf Beta-Lactam-Antibiotika, an eine Legionellen-Infektion gedacht werden. 
Tab. 1 Klinische Symptome der Legionärskrankheit (adaptiert nach Cunha, 2006)

\begin{tabular}{|c|c|c|c|}
\hline Organ & $\begin{array}{l}\text { Typische Symptome/ } \\
\text { Befunde }\end{array}$ & $\begin{array}{l}\text { Seltene Symptome/ } \\
\text { Befunde }\end{array}$ & $\begin{array}{l}\text { Gegen eine Legionellose } \\
\text { sprechende Symptome } \\
\text { /Befunde }\end{array}$ \\
\hline \multicolumn{4}{|l|}{ Klinische Symptome } \\
\hline ZNS & $\begin{array}{l}\text { Kopfschmerzen, } \\
\text { Verwirrtheit, Lethargie }\end{array}$ & Schwindel & Meningismus, Krampfanfälle \\
\hline $\mathrm{HNO}$ & Keine & Schwindel & $\begin{array}{l}\text { Hals-/ Ohrenschmerzen, } \\
\text { Otitis media }\end{array}$ \\
\hline Kardial & Relative Bradykardie & $\begin{array}{l}\text { Legionellen- } \\
\text { Endokraditis }\end{array}$ & Septische Embolien \\
\hline Gastrointestinal & wässrige Diarrhoe & Abdominalschmerzen & $\begin{array}{l}\text { Peritonismus, Druckdolenz } \\
\text { über der Leber }\end{array}$ \\
\hline Niere & Kreatinin $\uparrow$ & $\begin{array}{l}\text { Akutes } \\
\text { Nierenversagen }\end{array}$ & $\begin{array}{l}\text { Chronischen } \\
\text { Nierenversagen }\end{array}$ \\
\hline \multicolumn{4}{|l|}{ Labor } \\
\hline Liquor & Normal & Leichte Pleozytose & Lactat $\uparrow$ \\
\hline $\begin{array}{l}\text { Direktmikroskopie } \\
\text { Sputum }\end{array}$ & $\begin{array}{l}\text { Kein Nachweis von } \\
\text { Bakterien }\end{array}$ & $\begin{array}{l}\text { Wenige } \\
\text { mononukleäre Zellen, } \\
\text { Mischflora }\end{array}$ & $\begin{array}{l}\text { Purulentes Sputum mit } \\
\text { massenhaft } \\
\text { Erregernachweis }\end{array}$ \\
\hline Pleura & Exsudat & Transsudat & Empyem $(\mathrm{pH} \downarrow)$ \\
\hline Transaminasen & $<2 \times$ normal $\uparrow$ & $>2 x$ normal $\uparrow$ & $>10 x$ normal $\uparrow$ \\
\hline Urin & Mikrohämaturie & $\begin{array}{l}\text { Proteinurie, } \\
\text { Myoglobulinurie }\end{array}$ & $\begin{array}{l}\text { Makrohämaturie, Pyurie, } \\
\text { Hämoglobinurie }\end{array}$ \\
\hline
\end{tabular}

\section{Komplikationen}

Die Legionellen-Pneumonie zeigt häufig dann einen komplizierten Verlauf, wenn eine Immunsuppression vorliegt. Dabei wurden die meisten Formen von Krebserkrankungen,

Organtransplantation, Steroidtherapie und die Behandlung mit Tumornekrosefaktor alpha (TNF $\alpha$ )Antagonisten als Risikofaktoren identifiziert.

Der Grossteil dieser Patienten leidet unter hohem Fieber, Schüttelfrost und Husten sowie einer multilobären oder bilateralen Beteiligung. Nicht selten kommt es bei diesen Patienten auch zu Mehrfachinfektionen (z.B. GytomegalieZytomegalie-Virus Infektionen oder Nokardiose). Eine gefürchtete Komplikation ist die akute respiratorische Insuffizienz mit Intubationsbedürftigkeit und unter Umständen sogar letalem Verlauf.

Insgesamt ist der Verlauf einer Legionellen-Pneumonie in dieser Patientengruppe schwerer und eine rasche antibiotische Therapie dringend indiziert. Zum Beispiel besteht bei der Haarzell-Leukämie, welche zu einer Monozytopenie und Monozytendysfunktion führt, ein Defekt in der zellvermittelten Immunität. Diese Patienten haben ein erhöhtes Risiko für Infektionen durch Erreger, die unter der Kontrolle einer zellvermittelte Abwehr stehen (v.a. Legionellen). 


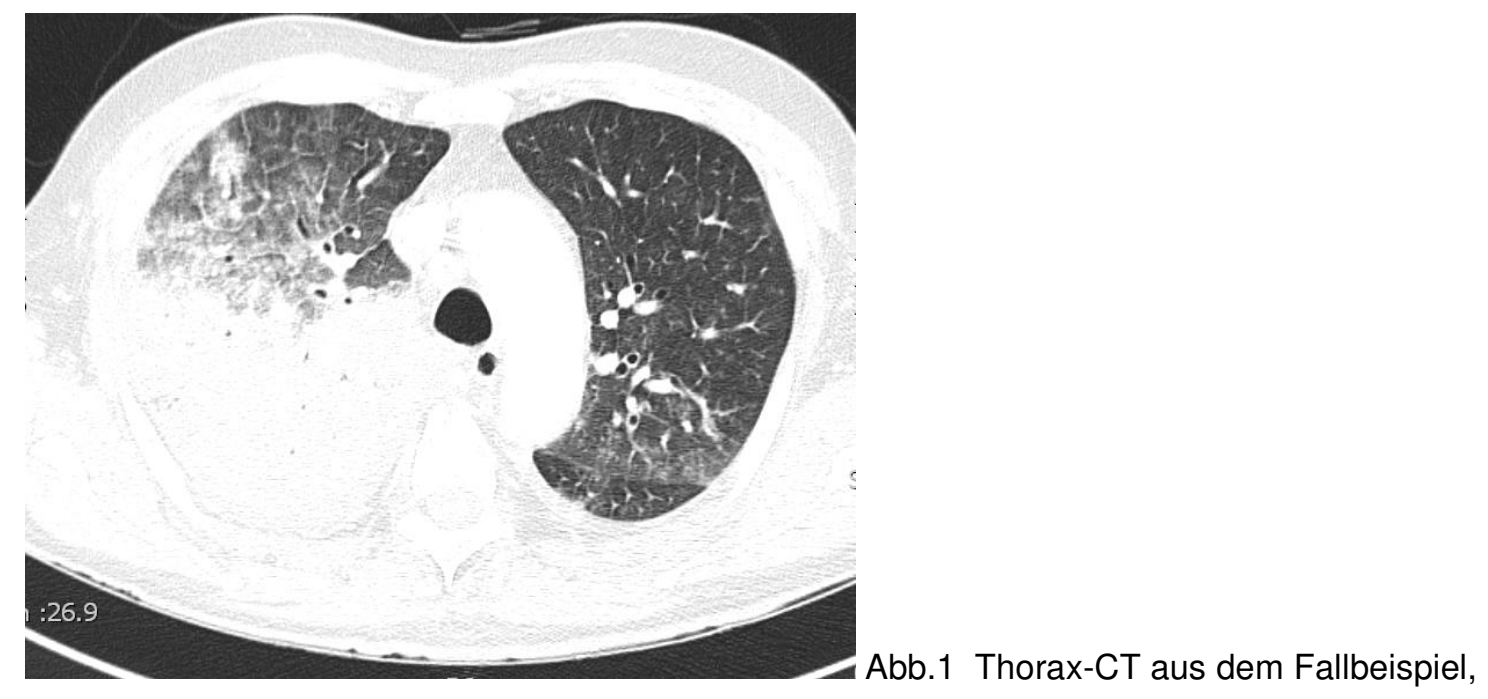

Lungenfenster. Ausgedehnte Konsolidation des rechten Oberlappens sowie beginnende

Transzparenzminderung im lateralen Mittellappen. 
Die Diagnose und Abklärung der Legionärs-Krankheit beinhaltet die Anamnese, Bildgebung, UrinAntigentest, differentialdiagnostische Überlegungen bis hin zu spezifischen Untersuchungen wie PCRNachweis mittels bronchoalveolärer Lavage (BAL). Aufgrund der unspezifischen klinischen Präsentation ist eine rasche Diagnosestellung und prompte Therapieeinleitung wichtig, da diese entscheidend das klinische Outcome verbessert.

Bei jedem Patienten mit hospitalisationsbedürftiger Pneumonie sollte routinemässig ein Legionellen Urin-Antigentest angeordnet werden. Dieser ist schnell und kostengünstig. Berücksichtigt werden muss dabei jedoch, dass dieser ausschliesslich die Serogruppe 1 erfasst, welche der häufigste klinisch relevante Serotyp ist. Bei klinischem Verdacht und negativem Urin-Antigentest sollten deshalb unbedingt weitere Abklärungen erfolgen. In diesem Fall sind eine Keimisolierung mittels Kultivierung und/oder eine DNA-Amplifikation mittels Legionellen-PCR (aus Sputum oder BAL) indiziert.

Die Hospitalisationsbedürftigkeit einer Pneumonie kann anhand verschiedener, gut validierter RisikoScores beurteilt werden. Sehr pragmatisch und einfach anwendbar ist der sogenannte CURB-65Score. Dieser ist ein Akronym, bei dem die einzelnen Buchstaben für die englisch bezeichneten klinischen Befunde bzw. Laborwerte stehen. Letztlich ist jedoch immer die klinische Gesamtbeurteilung entscheidend. Gerade bei Patienten mit zusätzlichen Erkrankungen tendiert der Score dazu, den Schweregrad zu unterschätzen.

Die Elemente des Akronyms stehen für folgende englische Bezeichnungen:

$$
\begin{aligned}
& C=\text { Confusion (Verwirrtheit) } \\
& U=\text { Urea (Serumharnstoff) } \\
& R=\text { Respiratory (Atmung) } \\
& B=\text { Blood pressure (Blutdruck) } \\
& 65=\text { Patient ist } \geq 65 \text { Jahre alt }
\end{aligned}
$$

\begin{tabular}{|c|c|c|}
\hline Symptome/Befunde & Punkte & Hospitalisationsindikation \\
\hline Neue Verwirrtheit & 1 & \multirow{5}{*}{$\begin{array}{l}\text { 0-1 Punkte: Ambulante Therapie } \\
2 \text { Punkte: Stationäre Therapie } \\
\text { 4-5 Punkte: IPS-Indikation evaluieren }\end{array}$} \\
\hline Harnstoff (Serum) $>7 \mathrm{mmol} / \mathrm{l}$ & 1 & \\
\hline Atemfrequenz $>30 / \mathrm{min}$ & 1 & \\
\hline Blutdruck SBD $<90$ und/oder DBD $<60 \mathrm{mmHg}$ & 1 & \\
\hline Alter $\geq 65$ Jahre & 1 & \\
\hline
\end{tabular}

Tab. 2 Hospitalisationsindikation nach dem CURB 65-Score 


\section{Ursachen, Diagnosekriterien und Differentialdiagnose}

\section{Ursachen}

Legionellen-Infektionen entstehen in der Regel durch Inhalation von entsprechend kontaminierten Aerosolen, welche durch Wassersysteme, wie Kühltürme, Klimaanlagen, Duschanlagen und Heisswasser-Anlagen produziert werden. Selten sind Aspirationen sowie die direkte Übertragung in die Lunge durch Eingriffe an den Atemwegen Ursachen für eine Infektion. Verschiedene Faktoren tragen zum Überleben und zur Vermehrung der Legionellen im warm-feuchten Milieu bei. Die Bildung eines Biofilms wird als wichtigster biologischer Faktor diskutiert.

\section{Diagnosekriterien und Differentialdiagnose}

Im klinischen Alltag ist es nicht immer einfach, eine Legionellen-Pneumonie von anderen PneumonieErregern zu unterscheiden. In manchen Fällen kann eine Legionellen-Pneumonie, welche sich in der Regel als sogenannte „atypische Pneumonie“ präsentiert, jedoch aufgrund ihrer klinischen Symptome identifiziert oder vermutet werden.

Atypische Pneumonien unterscheiden sich von den typischen insbesondere durch das Vorhandensein von extrapulmonalen Symptomen. Aufgrund dieser Feststellung wurde von Cunha 2006 eine einfache Stratifikation vorgeschlagen, welche eine differentialdiagnostische Hilfestellung (Abb.2) bei der Behandlung einer Pneumonie darstellen kann. Der Begriff „atypische“ Pneumonie ist jedoch keineswegs von allen Experten und Zentren akzeptiert. In diesem Zusammenhang sei darauf hingewiesen, dass eine schwere Sepsis oder ein septischer Schock im Rahmen einer PneumokokkenPneumonie ebenfalls extrapulmonale und somit „atypische“ Symptome aufweisen. Durchaus erwähnenswert erscheint jedoch, dass eine Legionelleninfektion häufig mit einer relativen Bradykardie einhergeht, womit gelegentlich eine Abgrenzung gegenüber anderen Pneumonie-Erregern vorgenommen werden kann. 
Abb. 2 Klinik und Erreger der ambulant erworbenen Pneumonie (adaptiert nach Cunha, 2006)

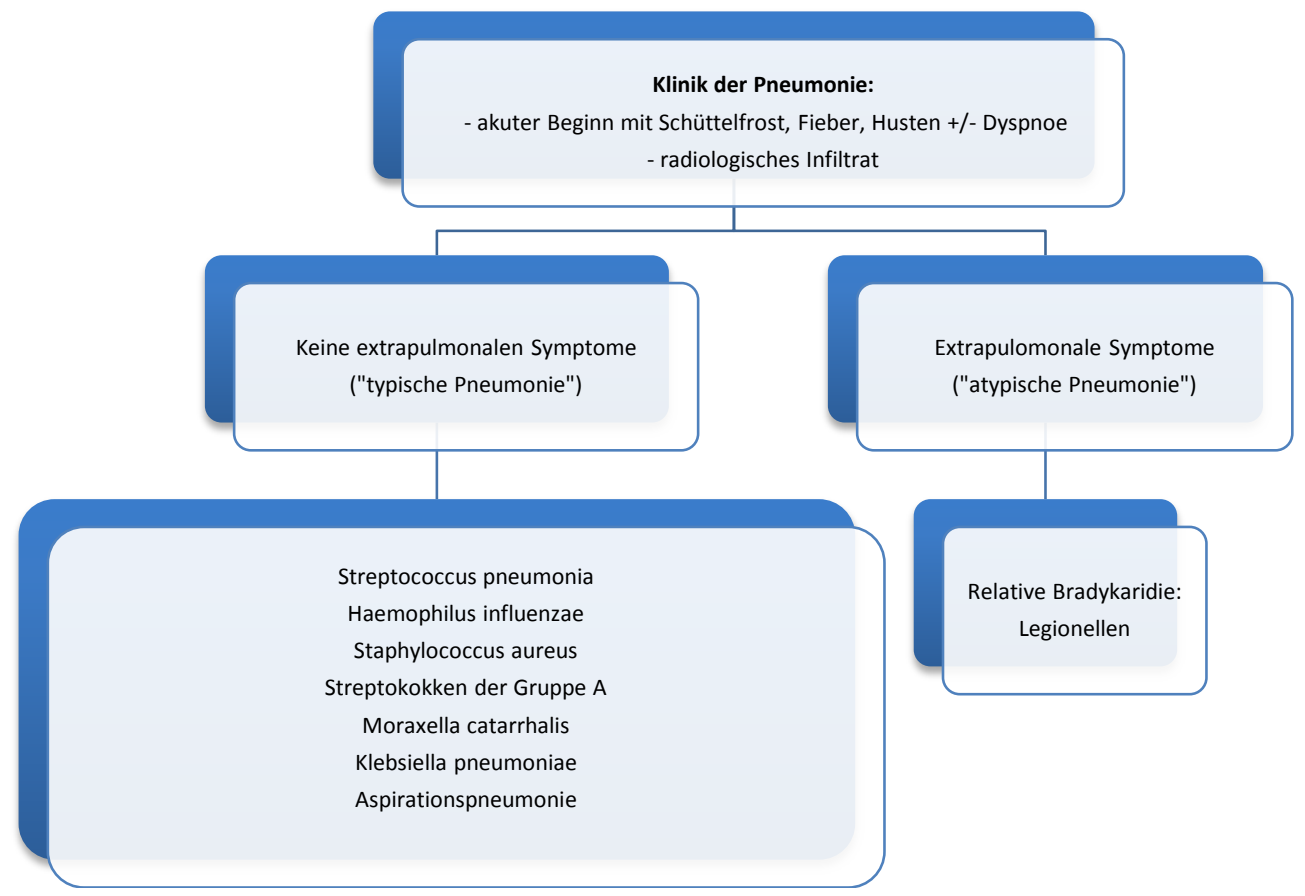




\section{Therapie}

Es sollte möglichst rasch eine effektive antibiotische Therapie etabliert werden, da eine unbehandelte oder ineffektiv therapierte Legionellen-Pneumonie eine Mortalität von 16-30\% aufweist. Bei immunkomprimierten Patienten liegt diese sogar bei bis zu 50\%. Adäquat und rechtzeitig behandelt kann die Mortalität dagegen auf $<10 \%$ gesenkt werden.

Bei der Wahl des Antibiotikums ist es wichtig, die Tatsache zu berücksichtigen, dass Legionellen als intrazelluläre Pathogene wirksam sind. Makrolide, Tetrazykline und Chinolone gehören zur Gruppe der intrazellulär wirksamen Antibiotika. Therapie der Wahl sind Fluorchinolone der neueren Generation (Levofloxacin, Moxifloxacin, Ofloxacin) und Makrolide (Clarithromycin, Azithromycin), wobei diese beiden ein vergleichbares klinisches Outcome aufweisen. Insgesamt sind bei den Fluorchinolonen jedoch ein rascherer Wirkungseintritt und weniger unerwünschte Arzneimittelnebenwirkung als auch eine höhere orale Bioverfügbarkeit als bei den Makroliden beschrieben.

Fluorchinolone der neueren Generation besitzen ein breites antibiotisches Wirkspektrum und sind auch gegen andere häufige Pneumonie-Erreger wie z.B. Streptococcus pneumoniae, wie auch gegen andere , atypische“ Erreger wie Mycoplasma pneumoniae und Chlamydia pneumoniae wirksam. Bei schwer verlaufenden, hospitalisationsbedürftigen Pneumonien soll hingegen neben einem Fluorchinolon oder Makrolid zusätzlich ein Betalaktamantibiotikum oder ein Cephalosporin verabreicht werden. Bei im Spital- oder in Pflegeeinrichtungen erworbenen Pneumonien ist aufgrund der aktuellen Resistenzlage (MRSA und multiresistente gram-negative Keime, z.B. ESBL) eine primäre Therapie mit Carbapenem (Meropenem) oder Piperacillin/Tazobactam empfohlen, wobei die Legionellen zusätzlich mit einem Fluorchinolon (z.B.Levofloxacin) oder mit einem Makrolid abgedeckt werden müssen. Levofloxacin ist ebenfalls bei transplantierten und HIV-Patienten die Therapie der Wahl, da Makrolide mit Immunsupressiva sowie mit antiretroviralen Medikamenten interagieren können.

Die Therapiedauer beträgt insgesamt 7-10 Tage. Bei immunsupprimierten oder kritisch kranken Patienten sollte die Therapiedauer auf 21 Tage verlängert werden.

\section{Prognose}

Bei immunkompetenten Patienten ist die Prognose der Legionärskrankheit mit einer Mortalität $<5 \%$ insgesamt gut. Vorrausetzung dafür ist die Etablierung einer raschen und effektiven antibiotischen Therapie. Ein verzögerter Therapiebeginn führt zu einem schlechteren Outcome, dies gilt insbesondere für immunsupprimierte Patienten. Zum Beispiel besteht bei der Haarzell-Leukämie, welche zu einer Monozytopenie und Monozytendysfunktion führt, ein Defekt in der zellvermittelten Immunität. Diese Patienten haben ein erhöhtes Risiko für Infektionen durch Erreger, die unter der Kontrolle einer zellvermittelte Abwehr stehen (v.a. Legionellen). 


\section{Prävention}

Die Legionärskrankheit wird nicht direkt von Mensch zu Mensch übertragen, so dass eine Isolation der Betroffenen nicht notwendig ist.

Da ein natürliches Vorkommen der aeroben, gram-negativen Bakterien Wasserreservoire sind, besteht die wichtigste Prävention, insbesondere in Spitälern und Pflegeinstitutionen, in einer regelmässigen Kontrolle des Wassers sowie der Wasserleitungen (Kupfer-Silber lonisierung). Dabei liegt die Temperatur für eine optimale Lebensbedingungen der Legionellen zwischen 25 und $50^{\circ} \mathrm{C}$, aus diesem Grund sollte bei Warmwassererzeugungsanlagen immer einer Temperatur von min. $60^{\circ} \mathrm{C}$ eingehalten werden. Bei Trinkwasser dagegen ist auf kühle Temperaturen zu achten. Eine Quellensuche bei Infektion sollte jeweils im Einzelfall in Erwägung gezogen werden. Bei einer Temperatur von mehr als $70^{\circ} \mathrm{C}$ werden die Legionellen innerhalb kürzester Zeit abgetötet, was für eine thermische Desinfektion genutzt werden kann. 


\section{Fallbericht}

Y

Jetziges Leiden: Die Zuweisung erfolgte bei unklarem septischem Zustandsbild, Inappetenz seit zwei Wochen sowie progredientem Ikterus. Zudem bestand zwei Tage vor Hospitalisation eine vollständige Nahrungsverweigerung. Auffallend in der Anamnese war zudem ein seit zwei Wochen intermittierend auftretendes Nasenbluten. Husten, Dyspnoe, Nachtschweiss oder Gewichtsverlust wurden verneint. Es bestanden keine gastrointestinalen Symptome.

Systemanamnese: Kein Nikotinabusus, regelmässiger Alkoholkonsum (3dl Bier/d). Keine regelmässige Medikamenteneinnahme

Persönliche Anamnese: blande bis auf Tonsillektomie in der Kindheit

Familienanamnese: Mutter 59-jährig an unbekannten Malignom verstorben

Sozialanamnese: Pensionierter Bauingenieur, verheiratet, 1 Sohn, 1 Tochter

\section{Weg zur Diagnose/Kommentar}

Auffallend ist, dass es bei diesem Patienten ohne bisherige Vorerkrankungen und ohne regelmässige Medikamenteneinnahme zu einer raschen und massiven Verschlechterung des Allgemeinbefindens ohne eigentlichen klinischen Fokus gekommen ist. 


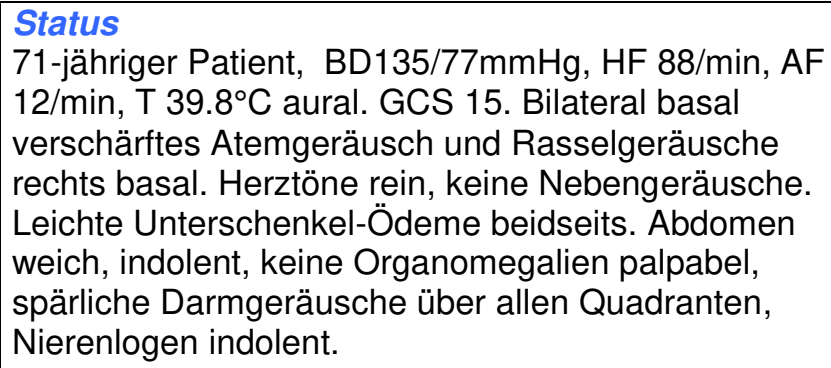

Alk.Phos.

Weitere Befunde

Legionellen-Antigen Urin negativ Pneumokokken Antigen Urin negativ CT Thorax: Pneumonische Konsolidationen im posterioren Oberlappen, apikalen, medio- und posterobasalen Unterlappen rechts

\section{Aktuelle Therapie}

Antibiotische Therapie mit Ceftriaxon (Rocephin $®)$

und Clarithromycin (Klazid $®$ ).

\section{Erweitertes Labor}

Arterielle Blutgasanalyse: respiratorische Globalinsuffizenz

Blutkulturen: kein Erregernachweis

Virologie: HCV, HIV, Influenza A/B, CMV, EBV, HSV negativ

Bronchoalveoläre Lavage(BAL): Legionellen PCR positiv

$\downarrow \quad(1.40-8.0 \mathrm{G} / \mathrm{l})$

$\downarrow$ (0.16-0.95 G/l)

$\uparrow(<1.2)$

$\downarrow(136-145 \mathrm{mmol} / \mathrm{l})$ $(3.3-4.5 \mathrm{mmol} / \mathrm{l})$

$\uparrow(<0.1 \mu \mathrm{g})$

$\uparrow(<21 \mu \mathrm{mol} / \mathrm{l})$

$(<50 \mathrm{U} / \mathrm{I})$

$\uparrow(10-50 \mathrm{U} / \mathrm{I})$

$\uparrow \uparrow \quad(40-129 \mathrm{U} / \mathrm{I})$
Weg zur Diagnose/Kommentar

$\mathrm{Zu}$ beachten ist die relative Bradykardie bei

febrilem Patienten

Im Routinelabor zeigten sich erhöhte Entzündungsparameter. Auffallend ist jedoch eine Monozytopenie. Desweiteren besteht eine Hepatopathie. In der Bildgebung zeigten sich dann ausgeprägte Infiltrate in der rechten Lunge.

Nach negativem Legionellen-Antigen im Urin wurde die antibiotische Therapie mit Clarithromycin gestoppt und nur mit Ceftriaxon fortgeführt. Unter dieser Monotherapie konnte jedoch keine Besserung der Pneumonie verzeichnet werden. Im weiteren Verlauf entwickelte der Patient dann eine rasch progrediente respiratorische Globalinsuffizienz und Hämoptoe, so dass eine Aufnahme auf die Intensivstation und mechanische Ventilation notwendig wurde. In der daraufhin erfolgten Bronchoskopie konnte schliesslich in der BAL Legionellen-spp.

mittels PCR nachgewiesen werden. 
Therapie des Patienten

Nach Diagnose der Legionellen-Pneumonie und Umstellung der antibiotischen Therapie auf ein Chinolon erholte sich der Patient weitgehend und konnte in die ambulante Therapie entlassen werden.

Bei Monozytopenie, Anämie und Thrombopenie konnte im Verlauf zytomorphologisch und in der Immunphänotypisierung eine Haarzell-Leukämie nachgewiesen werden, welche chemotherapeutisch weiterbehandelt wurde. Zu einer erneuten Pneumonie ist es nicht gekommen. Nach einem Follow up von neun Monaten ist der Patient weitgehend beschwerdefrei.

Insgesamt bestand bei diesem Patienten der dringende klinische Verdacht auf eine Legionellen-

Pneumonie. Aus diesem Grund hätte die antibiotische Therapie mit Clarithromycin fortgeführt und eine erweiterte Diagnostik mittels einer PCR-Untersuchung im Sputum oder in der BAL veranlasst werden müssen. Zudem gibt es Hinweise, dass bei schweren Pneumonieverläufen (insbesondere bei Pneumokokkenpneumonien) das Makrolid trotz negativen Legionellen-Antigen im Urin beibehalten werden sollte. 


\section{Fragen zur Legionellen-Pneumonie}

\section{Frage 1}

Welche Laborparameter sollten bei einem Patienten mit Symptomen einer Pneumonie an eine Legionellose denken lassen
a) Hyponatriämie und Hypophosphatämie
b) Hypernatriämie und Hyperphosphatämie
c) Eisenmangel
d) Hyperkalziämie
e) Erhöhte Kälte-Agglutinine

\section{Frage 2}

Therapie der Wahl bei Legionellose
a) Amoxicillin/Clavulansäure
b) Ciprofloxacin
c) Cephalosporine der 3. Generation
d) Levofloxacin
e) Sulfamethoxazol/Trimethoprim

\section{Frage 3}

Welche Serogruppen erfasst der Urin-Antigentest
a) alle
b) 1
c) 4 und 6
d) keine
e) 18 und 20

\section{Frage 4}

Bei welchen Patienten ist ein komplizierter Verlauf der Legionellose ungewöhnlich.
a) Organtransplantierte
b) Patienten unter Steroiden
c) Schwangere
d) Adoleszente
e) Patienten mit Stammzellerkrankungen

\section{Frage 5}

Bei welchen Symptomen sollte man an eine Legionellen-Erkrankung denken
a) Husten, Fieber und gastrointestinale Symptome
b) Husten, Fieber und Schüttelfrost
c) Husten, Fieber und Thoraxschmerzen
d) Husten, Fieber, Pharyngitis und Otitis
e) Husten, Fieber und Meningismus 


\section{Autoren}

Dr. med. Nadine Dobrick ${ }^{1}$, PD Dr. med. Nicolas Müller ${ }^{2}$, Dr. med. Daniel Franzen ${ }^{1}$

1 Klinik und Poliklinik für Innere Medizin, UniversitätsSpital Zürich

2 Klinik für Infektionskrankheiten und Spitalhygiene, UniversitätsSpital Zürich

\section{Korrespondenzadresse}

Dr. med. Nadine Dobrick

Klinik und Poliklinik für Innere Medizin

UniversitätsSpital Zürich

Rämistrasse 100

8052 Zürich

nadine.dobrick@usz.ch

\section{Bibliographie;}

Cunha BA: The atypical pneumonias: clinical diagnosis and importance. Clin Microbiol Infect 2006; 12 (Suppl.3):12-24.

Carratala J, Garcia-Vidal C: An update on Legionella. Curr Opin Infect Dis 2010; 23:152-157.

Woodhead M, Blase F, et al.: Guidelines for the management of adult lower respiratory tract infection; Eur Respir J 2005; 26: 1138-1180.

Barlow G, Nathwani D, Davey P. The CURB65 pneumonia severity score outperforms generic sepsis and early warning scores in predicting mortality in community-acquired pneumonia. Thorax 2007;62(3):253-9. 


\section{Antworten zu den Fragen zu Legionellose aus PRAXIS Nr. ?}

\section{Frage 1}

\section{Richtig ist Antwort a}

Ad $a$ und $b$ ) Eine Hyponatriämie und Hypophosphatämie kommt bei Pneumonien durch Legionellen häufiger vor als bei Pneumonien durch andere Erreger.

Ad $\mathrm{c}$ und d) Weder eine Hyperkalzämie noch ein Eisenmangel sind bei Pneumonien durch Legionellen bisher gehäuft beobachtet worden.

Ad e) Bei Patienten mit einer ambulant erworbenen Pneumonie, Symptomen der oberen Atemwege sowie einer Erhöhung der Kälte-Agglutinine (Titer > 1:64) sollte an eine Pneumonie durch Mycoplasma pneumoniae gedacht werden.

\section{Frage 2}

\section{Richtig ist Antwort d}

Levofloxacin (Tavanic $\left.{ }^{\circledR}\right)$ gehört zu der Gruppe der Fluorchinolone. Diese sind zusammen mit den Makroliden (z.B. Clarithromycin: Klacid®) Therapie der Wahl bei Pneumonien durch Legionellen, da diese intrazellulär wirksam sind. Bei den Chinolonen wird jedoch ein rascherer Wirkungseintritt und weniger unerwünschte Arzneimittelnebenwirkungen als bei den Makroliden beschrieben.

\section{Frage 3}

\section{Richtig ist Antwort b}

Es sind mehr als 70 Serogruppen der Legionellen-Familie bekannt, wobei die Serogruppen 1,4 und 6 für Humaninfektionen verantwortlich sind. Der Urin-Antigentest

Frage 4 auf Legionellen erfasst dabei ausschliesslich die Serogruppe 1.

\section{Richtig ist Antwort d}

Immunsupprimierte Patienten, wie organtransplantierte Patientin unter Steroidtherapie oder solche mit einer Stammzellerkrankung (z.B. die Haarzell-Leukämie) haben ein erhöhtes Risiko für einen komplizierten Verlauf der Legionellen-Pneumonie. In der Schwangerschaft besteht ebenfalls ein erhöhtes Risiko für einen komplizierten Verlauf

\section{Frage 5} der Legionellenpneumonie.

\section{Richtig ist Antwort a}

Ad a)

Bei Patienten mit ambulant erworbener Pneumonie und gastrointestinalen Symptomen, insbesondere wässrige Diarrhoe, sollte an eine durch Legionellen verursachte Pneumonie gedacht werden.

Ad b und c) Schüttelfrost und Thoraxschmerzen, - im Sinne von pleuritischen Schmerzen gehören zu den gängigen Symptomen einer Pneumonie, unabhängig vom Erreger.

Ad d) Diese Symptomkonstellation ist eher bei Pneumonien durch Mycoplasma pneumoniae zu erwarten.

Ad e) Typischerweise kommt es bei einer Pneumonie durch Legionellen zu Kopfschmerzen, Verwirrtheit und Lethargie. Das Vorliegen eines Meningismus spricht gegen eine Legionellose. 